\title{
THERMODYNAMICS OF PSEUDOSPIN-ELECTRON MODEL IN THE $U=0$ LIMIT
}

\author{
I.V. Stasyuk, A.M. Shvaika and K.V. Tabunshchyk \\ Institute for Condensed Matter Physics, National Academy of Sciences of Ukraine \\ 1 Svientsitskii Str., 290011 Lviv, Ukraine
}

Analytical consideration of a pseudospin-electron model in the absence of electron correlations is presented. Pseudospin and electron number mean values, thermodynamic potential, pair correlation functions are obtained in the same self-consistent approximation. The possibility of either first or second order phase transitions between different uniform phases (bistability) as well as between the uniform and the chessboard one is shown. In the regime $n=$ const, an instability with respect to phase separation can take place.

PACS numbers: 71.10.Fd, 71.38.+i, 77.80.Bh, 63.20.Ry

One of the models considering the interaction of electrons with local lattice vibrations is the pseudospin-electron model [1] in which local anharmonic variables are represented by pseudospins. Such model is used to describe the anharmonic vibrations of apex oxygen ions in $\mathrm{YBaCuO}$ type high $-T_{\mathrm{c}}$ superconductors (HTSC) [1].

The model Hamiltonian is the following:

$H=\sum_{i} H_{i}+\sum_{i j \sigma} t_{i j} b_{i \sigma}^{+} b_{j \sigma}, \quad H_{i}=U n_{i \uparrow} n_{i \downarrow}-\mu \sum_{\sigma} n_{i \sigma}+g \sum_{\sigma} n_{i \sigma} S_{i}^{z}-h S_{i}^{z} ;$

here, besides transfer the strong single-site electron correlations $U$ are included in the spirit of the Hubbard model and the subsystem of pseudospins placed in longitudinal field $h$ and interacting with conducting electrons ( $g$-term) is considered.

On the basis of pseudospin-electron model a possible connection between the superconductivity and the ferroelectric type instability in HTSC has been discussed $[2,3]$. An investigation of dielectric susceptibility of the model was performed in $[4,5]$ within the generalized random phase approximation (GRPA) [6] in the limit $U \rightarrow \infty$. The possibility of dipole and charge density modulation instabilities was shown. On the other hand, the case of $t_{i j}=0$ with the direct interaction between pseudospins was considered within the mean field approximation in [7]. The first or second order phase transitions with the jumps of $\left\langle S^{z}\right\rangle$ and electron concentration $n$ values in the $\mu=$ const regime were obtained. An instability with respect to phase separation in the electron and pseudospin subsystems can take place in the regime $n=$ const. 
In the absence of correlation $(U=0)$ operator (1) is close to the FalicovKimball (FK) model but differs in thermodynamic equilibrium conditions $\left(\left\langle S^{z}\right\rangle=\right.$ const for the FK model and $h=$ const for the pseudospin-electron one).

In the present work we propose for the case of the $U=0$ limit the self-consistent scheme for calculation of mean values of pseudospin and particle number operators, thermodynamic potential and correlation functions. The approach is based on the GRPA with the inclusion of the mean field corrections. The possibilities of phase separation and chessboard phase appearance are investigated.

The calculation is performed in the strong coupling case $(g \gg t)$ using single-site states as the basic one. The formalism of electron creation (annihilation) operators $a_{i \sigma}=b_{i \sigma} P_{i}^{+}, \tilde{a}_{i \sigma}=b_{i \sigma} P_{i}^{-}\left(P_{i}^{ \pm}=\frac{1}{2} \pm S_{i}^{z}\right)$ acting at a site with the certain pseudospin orientation is introduced. Expansion of the calculated quantities in terms of electron transfer leads to the infinite series of terms containing the averages of the $T$-products of the $a_{i \sigma}, \tilde{a}_{i \sigma}$ operators. The evaluation of such averages is made using the corresponding Wick theorem. The averages of the products of the projection operators $P_{i}^{ \pm}$are expanded in semi-invariants [8].

Nonperturbed electron Green function is equal to

$$
g\left(\omega_{n}\right)=\left\langle g_{i}\left(\omega_{n}\right)\right\rangle, \quad g_{i}\left(\omega_{n}\right)=\frac{P_{i}^{+}}{\mathrm{i} \omega_{n}-\varepsilon}+\frac{P_{i}^{-}}{\mathrm{i} \omega_{n}-\tilde{\varepsilon}},
$$

where $\varepsilon=-\mu+g / 2, \tilde{\varepsilon}=-\mu-g / 2$ are single-site energies. Single-electron Green function (calculated in Hubbard-I type approximation) is $\Rightarrow=G_{k}\left(\omega_{n}\right)=$ $\left[g^{-1}\left(\omega_{n}\right)-t_{k}\right]^{-1}$ and its poles determine the electron spectrum

$$
\varepsilon_{\mathrm{I}, \mathrm{II}}\left(t_{\boldsymbol{k}}\right)=\frac{t_{\boldsymbol{k}}}{2}-\mu \pm \frac{1}{2} \sqrt{g^{2}+4 t_{k}\left\langle S^{z}\right\rangle g+t_{\boldsymbol{k}}^{2}} .
$$

In the adopted approximation the diagrammatic series for the pseudospin mean value can be presented in the form

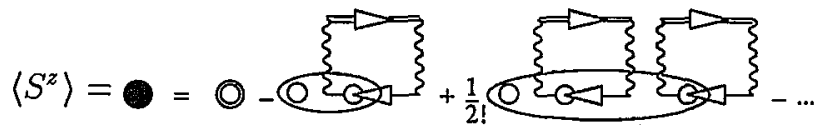

Here we use the following diagrammatic notations: $0-S^{z}, \longrightarrow-g_{i}\left(\omega_{n}\right)$, wavy line is the Fourier transform of hopping $t_{k}$. Semi-invariants are represented by ovals and contain the $\delta$-symbols on site indexes. In the spirit of the traditional mean field approach [8] the renormalization of the basic semi-invariant by the insertion of independent loop fragments is taken into account in (4).

The analytical expression for the loop is the following:

$$
\sum_{\bigotimes}=\frac{2}{N} \sum_{n, k} \frac{t_{k}^{2}}{g^{-1}\left(\omega_{n}\right)-t_{k}}\left(\frac{P_{i}^{+}}{i \omega_{n}-\varepsilon}+\frac{P_{i}^{-}}{\mathrm{i} \omega_{n}-\tilde{\varepsilon}}\right)=\beta\left(\alpha_{1} P_{i}^{+}+\alpha_{2} P_{i}^{-}\right) .
$$

It should be noted that within the self-consistent scheme of the GRPA, in the sequences of loop diagrams in the expressions for thermodynamic potential $\Omega$ and pair correlation functions $\left(\left\langle S_{i}^{z} S_{j}^{z}\right\rangle,\left\langle S_{i}^{z} n_{j}\right\rangle,\left\langle n_{i} n_{j}\right\rangle\right)$ the connections between any two loops by more than one semi-invariant are omitted.

From (4) and (5) follows the equation for pseudospin mean value

$$
\left\langle S^{z}\right\rangle=\frac{1}{2} \tanh \left\{\frac{\beta}{2}\left[h+\alpha_{2}\left(\left\langle S^{z}\right\rangle\right)-\alpha_{1}\left(\left\langle S^{z}\right\rangle\right)\right]+\ln \frac{1+\mathrm{e}^{-\beta \varepsilon}}{1+\mathrm{e}^{-\beta \tilde{\varepsilon}}}\right\} .
$$


The thermodynamic potential in the considered approximation has the form:

$$
\begin{aligned}
& \Delta \Omega=\Omega-\left.\Omega\right|_{t=0}=-\frac{2}{N \beta} \sum_{\boldsymbol{k}} \ln \frac{\left[\cosh \frac{\beta}{2} \varepsilon_{\mathrm{I}}\left(t_{\boldsymbol{k}}\right)\right]\left[\cosh \frac{\beta}{2} \varepsilon_{\mathrm{II}}\left(t_{\boldsymbol{k}}\right)\right]}{\left(\cosh \frac{\beta}{2} \varepsilon\right)\left(\cosh \frac{\beta}{2} \tilde{\varepsilon}\right)}+\left\langle S^{z}\right\rangle\left(\alpha_{2}-\alpha_{1}\right) \\
& -\frac{1}{\beta} \ln \cosh \left[\frac{\beta}{2}\left(h+\alpha_{2}-\alpha_{1}\right)+\ln \frac{1+\mathrm{e}^{-\beta \varepsilon}}{1+\mathrm{e}^{-\beta \tilde{\varepsilon}}}\right]+\frac{1}{\beta} \ln \cosh \left(\frac{\beta}{2} h+\ln \frac{1+\mathrm{e}^{-\beta \varepsilon}}{1+\mathrm{e}^{-\beta \tilde{\varepsilon}}}\right) .
\end{aligned}
$$

The solution of Eq. (6) and calculation of potential $\Omega$ (as well as of the pair correlation functions in GRPA) were performed numerically for the square lattice with nearest-neighbor hopping $(d=2$ DOS with the band width $2 W)$.

In the $\mu=$ const regime the stable states are determined from the minimum of the thermodynamic potential (Fig. 1). In the uniform case, there exists a possibility of the first order phase transition with the jump of the pseudospin mean value and reconstruction of the electron spectrum [8].
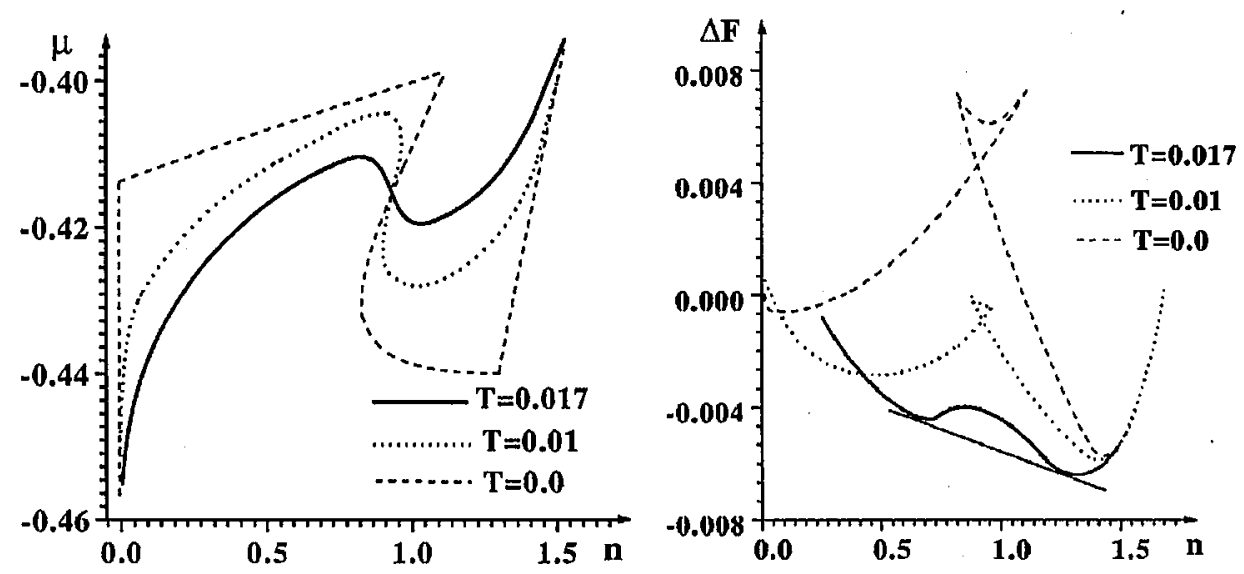

Fig. 1. Dependence of the chemical potential $\mu$ on the electron concentration $n$ and deviation of the free energy from linear dependence $\Delta F=F(n)-\left[\frac{n}{2} F(2)+\left(1-\frac{n}{2}\right) F(0)\right]$ for different $T$ values $(g=1, W=0.2, h=0.2)$.

In the $n=$ const regime the equilibrium condition is determined by the minimum of free energy $F=\Omega+\mu N$. One can see (Fig. 1) that the regions with $\mathrm{d} \mu / \mathrm{d} n \leq 0$, where states with a homogeneous distribution of particles are unstable, exist. This corresponds to the phase separation into the states with different electron concentrations and pseudospin mean values. In the phase separated region the free energy as a function of $n$ deflects up (Fig. 1) and concentrations of the separated phases are determined by the tangent line touch points.

The analysis of the $\left\langle S^{z} S^{z}\right\rangle_{q}$ correlator temperature behaviour shows that the high temperature phase becomes unstable with respect to fluctuations with $q \neq 0$ for certain values of model parameters. The maximal temperature of instability is achieved for $q=0$ or $q=(\pi, \pi)$ and indicates the possibility of phase transition into amodulated (chessboard) phase.

The analytical consideration of the chessboard phase can be performed in a similar way. 


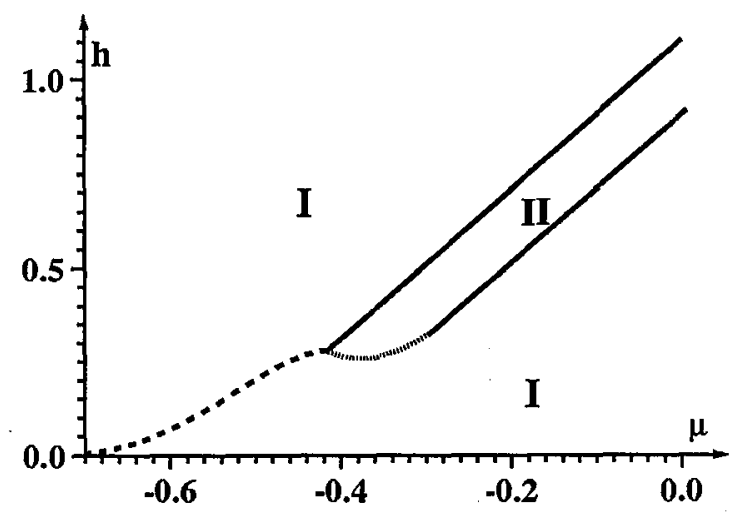

Fig. 2. $\quad \mu-h$ phase diagram $(T=1 / 80, W=0.2, g=1)$. I - uniform phase, II modulated phase.

From the comparison of the thermodynamic potential $\Omega$ values for uniform and chessboard phases, the $(\mu, h)$ phase diagram is obtained (Fig. 2). One can see that chessboard phase exists as intermediate one between the uniform phases with different $\left\langle S^{z}\right\rangle$ and $n$ values. The transition between different uniform phases (bistability) is of the first order (Fig. 2, dashed line), while the transition between the uniform and modulated ones is of the first (dotted line) or second (solid line) order.

Appearance of various phases in the considered model reminds the situation known for the FK model with a rich phase diagram [9]. However, contrary to this model, an existence of uniform phases is possible in our case. This results from another regime of thermodynamic averaging (fixation of $h$ field which is analogous to the chemical potential in the FK model).

\section{References}

[1] K.A. Müller, Z. Phys. B 80, 193 (1990).

[2] J.E. Hirsch, S. Tang, Phys. Rev. B 40, 2179 (1989).

[3] M. Frick, W. von der Linden, I. Morgenstern, H. Raedt, Z. Phys. B 81, 327 (1990).

[4] I.V. Stasyuk, A.M. Shvaika, Acta Phys. Pol. A 85, 363 (1994).

[5] I.V. Stasyuk, A.M. Shvaika, O.D. Danyliv, Mol. Phys. Rep. 9, 61 (1995).

[6] Yu.A. Izyumov, B.M. Letfulov, J. Phys., Condens. Matter 2, 8905 (1990).

[7] I.V. Stasyuk, Yu. Havrylyuk, preprint of the Institute for Condensed Matter Physics, ICMP-98-18E, Lviv 1998, p. 20.

[8] I.V. Stasyuk, A.M. Shvaika, K.V. Tabunshchyk, Cond. Matter Phys. 2, 109 (1999).

[9] J.K. Freericks, Phys. Rev. B 47, 9263 (1993). 